\title{
ПРИМЕНЕНИЕ ПЛАМЕННО-ИОНИЗАЦИОННОГО ДЕТЕКТОРА ДЛЯ КОЛИЧЕСТВЕННОГО ОПРЕДЕЛЕНИЯ ПРИМЕСЕЙ В ОРГАНИЧЕСКИХ СОЕДИНЕНИЯХ
}

Для определения степени чистоты органических соединепий часто применяют газовую хроматографию [1-4]. По сравнению с другими методами определения чистоты вецеств, она имеет преимущества, главным образом, в скорости, чувствительности и универсальности. В случае применения специальных обогатительных методов при помощи газовой хроматографии можно определять от $10-7$ до $10^{-12}$ доли примесей в исследуемом веществе $\left[{ }^{5-12}\right]$. Но иногда обогащение невозможно или нецелессобразно. В частности, при анализе соединений, очищенных при помощи препаративной газовой хроматографии, приходится определять.самые различные примеси, структуры которых часто известны только приблизительно. Содержание примесей составляет обычно от 10-2 до 10-6 доли. Вследствие их сложного состава методы обогащения обычно ше могут быть применены. В таких случаях необходимо пользоваться детектором высокой чувствительности, например детектором ионизационного типа. Так как чувствительность ионизационных детекторов зависит от анализируемых веществ, количественная интерпретация получаемых хроматограмм затруднена.

Первые описания пламенно-ионизационных детекторов приведены в трудах Дж. Мак-Вильяма и Р. Дюара $[13-15]$ в 1957 г. Дж. Хэрли и др. [16] в 1958 г. Уже в этих работах обсуждается сущность ионизационных процессов в детекторе. Но более глубоко этим вопросом начали заниматься лишь тогда, когда пламенно-ионизационные детекторы стали использоваться в количественном анализе.

Работа пламенно-ионизационного детектора основывается на увеличении проводимости пламени водорода при поступлении в него органического вещества. Так как ионизационные потенциалы органических молекул имеют значения от 9 до 12 эв, а фактически наблюдаемое увеличение проводимости близко к ионизационному потеіциалу элементарного углерода $(4,6$ эв), иоңизацию в пламени нельзя объяснять только на основе термического разложения.

Штерн $[17,18]$ предложил, что пламя начинает обладать проводимостью вследствие. возникающих в нем при горении агрегатов углерода. Поскольку теория агрегатов углерсда не может объяснить многие явления в детекторе, Х. Қалкот $[19,20]$ высказал мнение, что ионизация проявляется под действием освободившейся энергии экзотермиче ских реакций, происходящих в пламени.

Различие в ионизации для разных веществ ведет к различию в чувствительності детектора к этим веществам.

В работах, посвященных этому вопросу, сравниваются чувствительности детектора к разным вешествам, а также приводятся системы инкрементов для их вычисления $[21-27]$.

Так как мы располагали ограниченным количеством сведений по удельной чувствнтельности органических соединений и их классов, для целей настоящей работы необходимо было их дополнить и, в частности, рассмотреть вопрос с точки зрения количественного анализа неизвестных примесей. 
Аппаратура. По мнению ряда авторов [21] чувствительность пламенноионизационного детектора настолько сильно зависит от его конструкции и режима работы, что нельзя сравнивать данные, полученные на разных

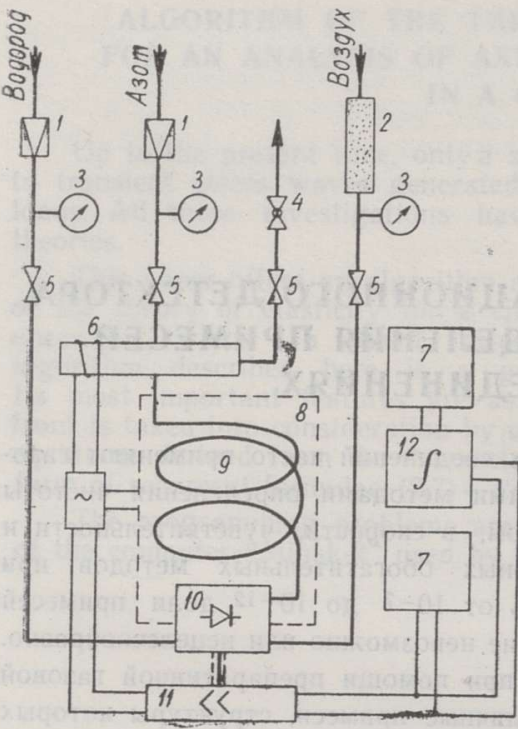

Рис. 1. Схема хроматографа FB-4: $i$ редуктор; 2 - фильтр; 3 - манометр; 4 -кран; 5 - вентиль; 6 - испаритель-распределитель; 7 - самописец; 8 - термостат; 9 - колонка; 10 детектор; 11 - усилитель; 12 - интегратор.

приборах. Однако удельные чувствительности, полученные разными авторами на разных приборах при оптимальных режимах работы детекторов, достаточно близки между собой. Следовательно, для получения сравнимых данных необходимо тщательно подбирать условия эксперимента.

В настоящей работе применялся газовый хроматограф FB-4 фирмы «Шендон». К прибору дополнительно были подключены интегратор [28] и фильтр воздуха. Схема аппаратуры приведена на рис. 1.

Сначала были исследованы характеристики усилителя сигнала детектора при минимальном и максимальном входном сопротивлении. Результаты показали, что участок прямолинейности позволяет интегрировать пики хроматограмм, высота которых не превышает двукратной ширины ленты самописца при минимальной чувствительности.

В качестве газа-носителя применяли азот. Водород и воздух поступали в детектор отдельно. Так как от скорости газа-носителя зависит разделяющая способность колонки, величина ее была выбрана оптимальной для применяемой колонки. Для колонки диаметром 2 мм и длиной 4 м с насадкой, пропитанной полиэтиленгликолем, скорость газа-носителя, равная $10 \mathrm{~m} / \mathrm{muн}$, оказалась оптимальной.

После этого были определены оптимальные скорости водорода и воздуха, соответствующие выбранной скорости потока газа-носителя. При разных скоростях водорода и температуре колонки $72^{\circ} \mathrm{C}$ в колонку вводился бензол по 0,5 мкл. Площади пиков интегрировали. Данные приведены на рис. 2.

Оказалось, что чувствительность детектора зависит от скорости потока водорода. При

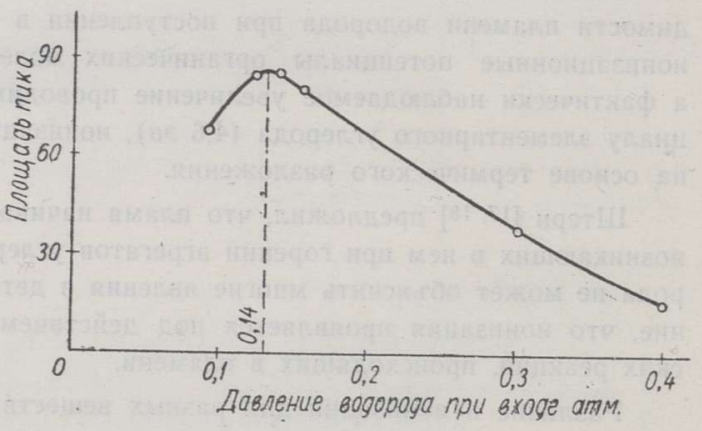

Рис. 2. Зависимость чувствительности детектора от скорости водорода. этом, как видно из графика, в пределах от 0,11 до 0,17 атм имеется максимум, вблизи которого чувствительность детектора слабо зависит от колебаний скорости потока водорода.

Оптимальным давлением воздуха оказалась 1 атм.

Была определена также зависимость чувствительности детектора от 
напряжения электродов. Оказалось, что при нормальной скорости газа чувствительность практически не меняется в пределах от 300 до 1200 в.

Линейность показаний детектора. Линейная зависимость между площадью пика и количеством пробы при применении пламенно-ионизационного детектора известна. Однако эта линейность доказана для ограниченного диапазона концентраций вещества в газе-носителе.

Несмотря на то, что пределы линейности связаны с концентрацией, их часто, в целях наглядности, выражают через максимальное количество введенной пробы. При этом, однако, надо иметь в виду, что одинаковые количества проб могут давать разные максимальные концентрации. Более концентрированный выход жомпонента получается при хроматографировании однокомпонентных веществ с малым объемом удерживания в коротких колонках, где

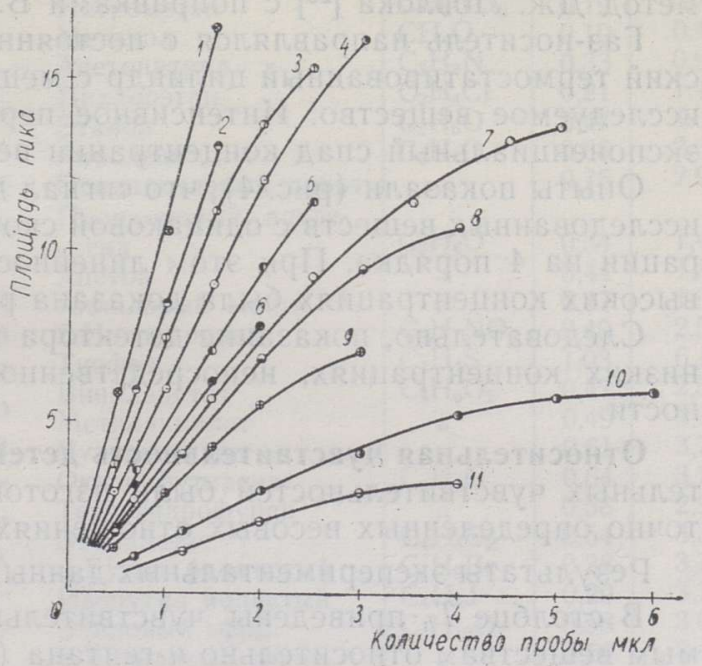

Рис. 3. Зависимость между площадью пика и количеством пробы: 1 - лимонен; 2 - этилбензол; 3 - н-октан; 4 - н-гептан; 5 - этнлацетат; 6 - этиловый эфир; 7 - этанол; 8 - ацетон; 9 - ацетонитрил; 10 - метанол; 11 - трихлорметан. смывание вещества за счет разделения на компоненты и за счет продольной диффузии мало.

Опыты, проделанные нами с разными веществами, показали, что при выбранных условиях эксперимента объем вводимой пробы не должен перевышать 2 мкл (рис. 3 ).

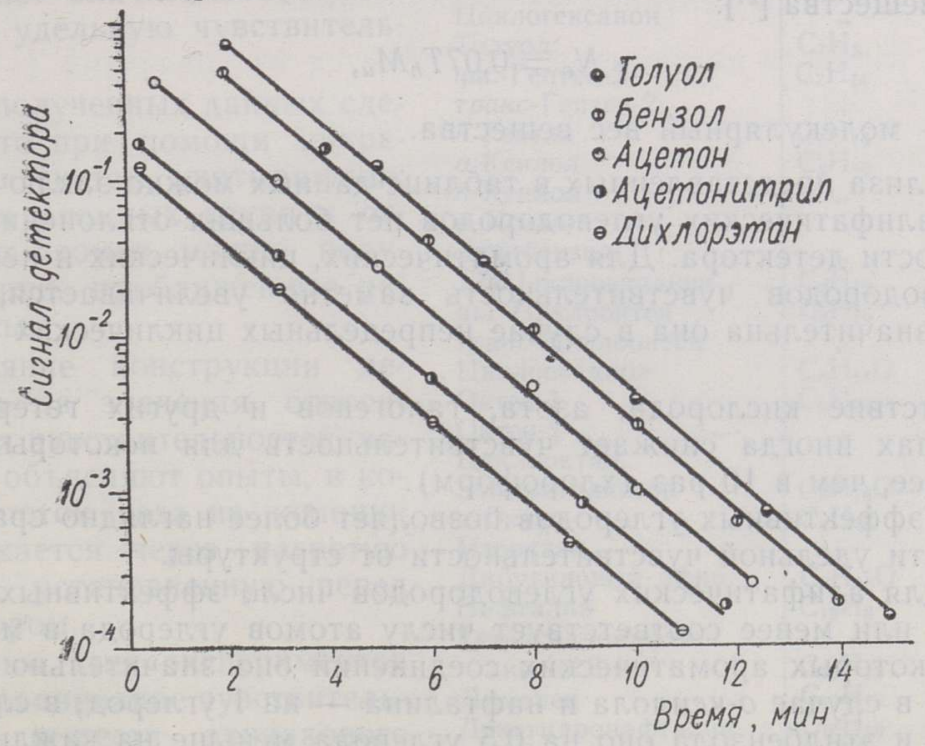

Рис. 4. Характеристики пламенно-ионизационного детектора. 
При определении микропримесей в пробе особый интерес представляет вопрос линейности в области концентраций непосредственно вблизи нижней границы чувствительности детектора.

Для изучения линейности при низких концентрациях был применен метод Дж. Ловлока $\left.{ }^{29}\right]$ с поправками В. Калмановского [22].

Газ-носитель направлялся с постоянной скоростью через металлический термостатированный цилиндр с мешалкой, в который было введенс исследуемое вещество. Интенсивное перемешивание газа обеспечивало экспоненциальный спад концентрации вещества в потоке газа-носителя.

Опыты показали (рис. 4), что сигнал детектора уменьшается для всех исследованных веществ с одинаковой скоростью при понижении концентрации на 4 порядка. При этом линейность детектора при относительно высоких концентрациях была доказана ранее (см. рис. 3).

Следовательно, показание детектора можно считать линейным и при низких концентрациях, непосредственно вблизи границы чувствительности.

Относительная чувствительность детектора. Для определения относительных чувствительностей были изготовлены смеси ряда веществ в точно определенных весовых отнөшениях. Стандартом служил н-гептан.

Результаты экспериментальных данных приведены в таблице (стр. 41).

В столбце $T_{h}$ приведены чувствительности детектора к анализируемым веществам относительно $н$-гептана (чувствительность которого принята за единицу):

$$
T_{h}=\frac{S_{u} m_{h}}{S_{h} m_{u}}
$$

где $S_{u}-$ площадь пика исследуемого вещества;

$S_{h}$ - площадь пика н-гептана;

$m_{u}$ - вес исследуемого вещества в смеси;

$m_{h}$ - вес н-гептана в смеси.

В столбце $N_{c}$ приведены так наз. числа эффективных углеродов для каждого вещества $\left[{ }^{21}\right]$ :

$$
N_{c}=0,07 T_{h} M_{u},
$$

где $M_{u}$ - молекулярный вес вещества.

Из анализа представленных в таблице данных можно заключить, что в случае алифатических углеводородов нет больших отклонений в чувствительности детектора. Для ароматических, циклических и непредельных углеводородов чувствительность заметно увеличивается, причем особенно значительна она в случае непредельных циклических соединений.

Присутствие кислорода, азота, галогенов и других гетероатомов в молекулах иногда снижает чувствительность для некоторых соединений более, чем в 10 раз (хлороформ).

Число эффективных углеродов позволяет более наглядно сравнивать. зависимости удельной чувствительности от структуры.

Если для алифатических углеводородов число эффективных углеродов более или менее соответствует числу атомов углерода в молекуле, то для некоторых ароматических соединений оно значительно больше, например, в случае a-ксилола и нафталина - на 1 углерод; в случае же $n$-ксилола и этилбензола оно на 0,5 углерода меньше на каждый цикл.

Аналогичное увеличение чувствительности наблюдается и в случае 
непредельных соединений, однако встречаются такие вещества, для которых число эффективных углеродов меньше числа углеродных атомов в молекуле (цис-гептен-2).

Одновременное присутствие непредельных связей и циклов сопровождается особенно сильным увеличением чувствительности (лимонен, циклооктен) .

Несколько точнее можно оценить влияние гетероатомов и функциональных групп. Для алифатических спиртов число эффективных углеродов на $0,5-0,6$ меньше, чем число углеродных атомов, для галогенов - на $0,3-0,4$, для простых эфиров, в том числе циклических, - на 0,3-1,0 и для алифатических нитросоединений на $0,2-0,5$ меньше. Қарбонильная группа в кетонах, альдегидах и сложных эфирах снижает чувствительность .. ңа $1-1,5$ углерода.

Удельные чувствительности для различных изомеров с одинаковым составом сильно отличаются друг от друга. Даже пространственная изомерия оказывает значительное влияние на удельную чувствительность.

Из полученных данных следует, что при помощи инкрементных систем, учитывающих влияния атомных групп и отдельных атомов, можно получить лишь приближенные результаты.

Влияние конструкции детектора на значения относительных чувствительностей частично объясняют опыты, в которых поток газа из колонки пропускается через нагретую трубку, установленную перед детектором.

Целью этих экспериментов было уравнивание чувствительностей веществ одинакового состава.

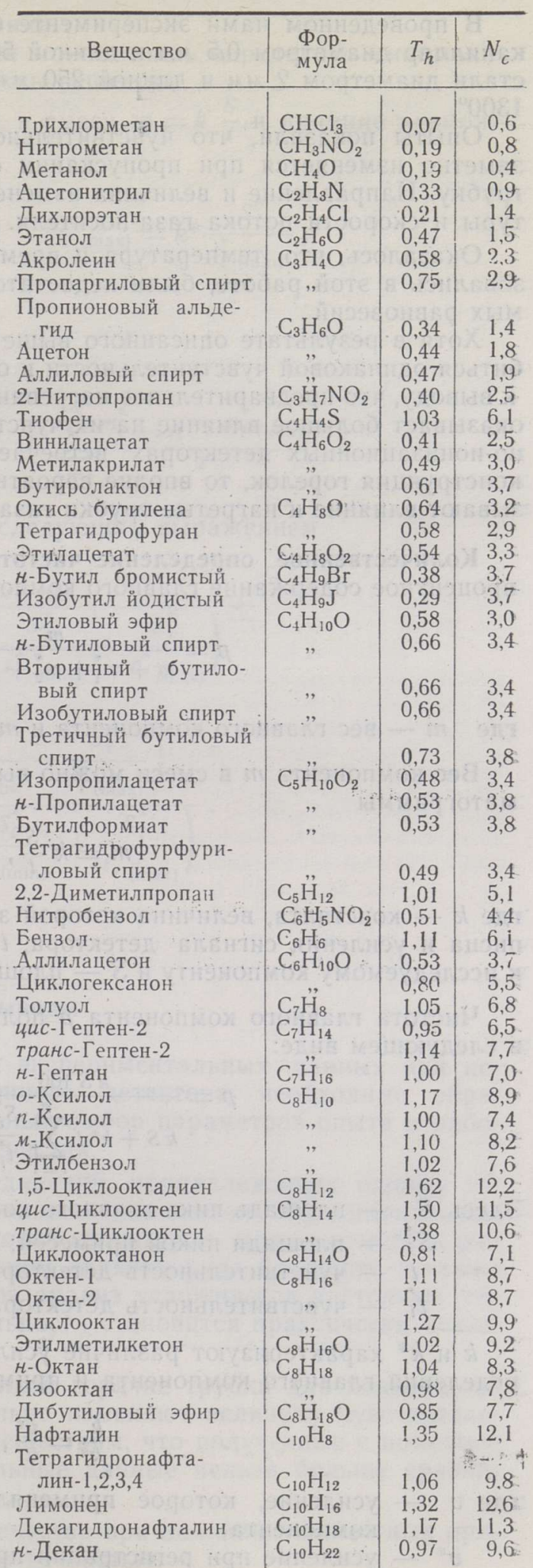


В проведенном нами эксперименте были использованы платиновый капилляр диаметром 0,5 мм и длиной 50 мм и трубка из нержавеющей стали диаметром 2 мм и длиной 250 мм, которые нагревались до $600-$ $1300^{\circ}$.

Опыты показали, что чувствительность к отдельным компонентам заметно изменяется при пропускании смесей веществ через нагретую трубку. Направление и величина изменений сильно зависят от температуры и скорости потока газа-носителя.

Оказалось, что температура и время нагревания, которые использовались в этой работе, были недостаточны для достижения необходимых равновесий.

Хотя в результате описанного выше эксперимента и не удалось добиться одинаковой чувствительности к отдельным веществам, он привел к выводу, что предварительное нагревание вводимых в детектор веществ оказывает большое влияние на их чувствительности. Так как в пламенно-ионизационных детекторах встречается различное расположение и жонструкция горелок, то вполне вероятно, что на чувствительности оказывают влияние и нагретые трубки газа-носителя.

Количественное определение чистоты. Чистотой вещества считают процентное содержание главного компонента в смеси:

$$
p=\frac{m}{m+m_{1}^{*}+m_{2}^{*}+\ldots m_{n}^{*}} 100 \%,
$$

где $m$ - вес главного компонента и $m_{1}^{*}, m_{2}^{*} \ldots m_{n}^{*}$ - веса примесей.

Вес компонента $m$ в смеси можно выразить через площадь пика хроматограммы

$$
m=k \frac{s}{t}
$$

где $k$ - константа, величина которой зависит от скорости ленты самописца и усиления сигнала детектора, $t$ - чувствительность детектора к исследуемому компоненту и $S$ - площадь пика.

Чистота главного компонента $p$ получается из уравнений (1) и (2) в следующем виде:

$$
p=\frac{k S 100}{k S+k^{*} t \sum_{i=1}^{n} \frac{S_{i}^{*}}{t_{i}^{*}}} \%
$$

Здесь $S$ - площадь пика главного компонента;

$S_{i}^{*}$ - площади пиков примесей;

$t$ - чувствительность детектора к главному компоненту;

$t_{i}^{*}$ - чувствительность детектора к примесям;

$k$ и $k^{*}$ характеризуют различие усилений сигнала детектора при определении главного компонента и примесей

$$
\frac{k}{k^{*}}=\frac{v^{*}}{v},
$$

где $v$ - усиление, которое применялось при регистрации главного компонента;

$v^{*}$ - усиление при регистрации примесей. 
Скорость ленты самописца постоянна.

В случае неизвестных примесей можно лишь определить минимальное и максимальное значение чистоты вещества.

Если вес главного компонента в смеси $m=k \frac{S}{t}$ и крайние возможные количества примесей

$$
m_{i(\min )}^{*}=k^{*} \frac{S_{i}^{*}}{t_{i(\max )}^{*}} \text { и } \quad m_{i(\max )}^{*}=k^{*} \frac{S_{i}^{*}}{t_{i(\min )}^{*}},
$$

где $i=1 \ldots n$,

то средняя доля примесей в смеси

$$
\sum_{i=1}^{n} \frac{m_{i(\max )}^{*}+m_{i(\min )}^{*}}{2} \pm \sum_{i=1}^{n} \frac{m_{i(\max )}^{*}-m_{i(\min )}^{*}}{2}
$$

и чистота вещества определяется следуюгцим выражением:

$$
\begin{gathered}
p=\frac{k S 100}{k S+\frac{k^{*} t}{2} \sum_{i=1}^{n}\left(\frac{S_{i}^{*}}{t_{i(\min )}^{*}}+\frac{S_{i}^{*}}{t_{i(\max )}^{*}}\right)} \pm \\
\pm \frac{\frac{k^{*} t}{2} \sum_{i=1}^{n}\left(\frac{S_{i}^{*}}{t_{i(\min )}^{*}}-\frac{S_{i}^{*}}{t_{i(\max )}^{*}}\right)}{k S+\frac{k^{*} t}{2} \sum_{i=1}^{n}\left(\frac{S_{i}^{*}}{t_{i(\min )}^{*}}+\frac{S_{i}^{*}}{t_{i(\max )}^{*}}\right)} .
\end{gathered}
$$

\section{Выводы}

1. Для получения сравнимых экспериментальных данных при использованин пламенно-ионизационного детектора необходимо обращать особое внимание на правильный выбор параметров опыта и работать на линейном участке характеристик.

2. Для чувствительностей соединений, принадлежащих одному гомологическому ряду или содержащих одинаковые функциональные группы, либо обладающих одинаковой структурой, можно отметить ряд закономерностей. При одновременном влиянии ряда факторов, а также при наличии неизвестных факторов анализ усложняется настолько, что предсказание значения чувствительности становится практически невозможным.

3. Расположенные перед горелкой горячие трубки для подвода газа-носителя иногда настолько сильно изменяют величину чувствительности детектора к органическим вецествам, что полученные с помощью разных детекторов экспериментальные данные нельзя больше сравнивать между собой.

4. Оценить степень чистоты вещества при наличии неизвестных примесей можно лишь с ограниченной точностью. 


\section{Л ИТЕ Р А Т У Р А}

1. Gi 11 M., Preston T., Jr., J. Gas Chromatog., 2 (12), 391 (1964).

2. N u s s G. R., Gas Chromatography, Instr. Soc. Am. Symposium - June 1963, Ed. by L. Fowler, Academic Press, New York, 1963, p. 119.

3. Huyten F. H., Rijnders G. W. A., van B e ersum W., Gas Chromatography 1962, Ed. by M. van Swaay, Butterworths, Washington, 1962, p. 335.

4. С акодынский К. И., Хохлова Л. А., Бра жников В. В., Сев рюго в а Н. Н., Газовая хроматография, Сб. статей I, изд. НИИТЭХИМ, М., 1964, стр. 96.

5. Eggersten F. T., Nels en F. H., Anal. Chem., 30 (6), 1040 (1958).

6. Brenner N., Ettre L. S., Anal. Chem., 31 (11), 1815 (1959).

7. Andre a tch A. J., Fe in 1 a nd R., Anal. Chem., 32 (8), 1021 (1960).

8. Feinland R., Andreatch A. J., Cotrupe D. P., Anal. Chem., 33 (8), 991 (1961)

9. B e nnet C. E., Nogare D. S., S a franski L. V., Lew is C. D., Anal. Chem., 30 (5), 898 (1958).

10. Davis A., Ro a 1 d i A., Tufts L. E., J. Gas Chromatog., 2 (9), 306 (1964).

11. Т уркельт а у 6 Н. М., Газовая хроматография, Тр. II Всесоюзн. конференции, M., 1964, стр. 88 .

12. S cott C. G., Phillip s C. S. G., Nature, 199 (4888), 66 (1963).

13. Mc Will i a m J. G., D ew a r R. A., Anal. Chem., 29 (6), 925 (1957).

14. M c Wi 11 i a m J. G., D e w a r R. A., Gas Chromatography 1958, Ed. by D. H. Desty, Butterworths Sci. Publ., London, 1958; p. 142.

15. M c W i 11 i a m J. G., D e w a r R. A., Nature, 181 (4611), 760 (1958).

16. H a r le y J., N e 1 W., Pretorius V., Nature, 181 (4603), 177 (1958).

.17. G a y don A. G., Wolf ha rd H. G., Flames, their Structure, Radiation and Temperature, Publ. Chapman \& Hall, London, 1953, p. 299.

18. L e w is B., E 1 b e G., Combustion, Flames and Explosions of Gases, Academic Press, New York, 1951.

19. C a $1 \mathrm{c}$ ot e H. F., Combustion and Flame, 1, 385 (1957).

20. Calcote H. F., King I. R., Fifth Symposium on Combustion, Reinhold Publ., New York, 1955, p. 423.

21. Sternberg J. C., G a 11 aw a y W. S., Jones D. T. L., Gas Chromatography, Instr. Soc. Am. Symposium - June 1961, Ed. by N. Brenner, J. E. Callen, M. D. Weiss, Academic Press, New York, 1962, p. 231.

22. К а л м а н в ски й В. И., Молекулярная хроматография, Сб. статей, М., 1964, стр. 22.

23. $M \mathrm{c} \mathrm{W}$ ill i a m J. G., J. Chromatog., 6 (3), 110 (1961).

24. Perkins G., Roua yheb G. M., Lively L. D., Ha milton W. C., Gas Chromatography, Instr. Soc. Am. Symposium - June 1961, Ed. by N. Brenner, J. E. Callen, M. D. Weiss, Academic Press, New York, 1962, p. 269.

25. Cohen E. N., P a r z en E., B a ile y D. M., J. Gas Chromatog., 1 (8), 14 (1963).

26. Halász I., S chneider W., Gas Chromatography, Instr. Soc. Am. Symposium June 1961, Ed. by N. Brenner, J. E. Callen, M. D. Weiss, Academic Press, New York, 1962 , p. 287.

27. E t t r e L. S., Gas Chromatography, Instr. Soc. Am. Symposium - June 1961, Ed. by N. Brenner, J. E. Callen, M. D. Weiss; Academic Press, New York, 1962, -p. 307.

28. С юг и с А., См. настоящий номер журнала, стр. 46.

29. Ловелок Дж., Газовая хроматография, Тр. III Международного симпозиума в Эдинбурге, М., 1964, стр. 27.

Институт кибернетики Академии наук Эстонской ССР
Поступила в редакцию

2/XI 1965 


\section{P. LUIGA, R. PUSS}

\section{LEEKIONISATSIOONDETEKTORI KASUTAMINE LISANDITE KVANTITATIIVSEL MÄARAMISEL ORGAANILISTES UHENDITES}

Kasutades ainete kvantitatiivseks gaasikromatograafiliseks uurimiseks leekionisatsioondetektorit, on vaja katseparameetrid valida täpselt. Tuleb töötada gaaside kiiruste, elektroodide pinge ja sisseantava ainekoguse võimalikult lineaarsetes piirkondades. Artiklis käsitletakse optimaalsete piirkondade valimise meetodeid ja antakse piirkondade sunrused kromatograafile FB-4. Näidatakse, et detektori tundlikkus erinevate orgaaniliste ainete suhtes sōltub üldjoontes ühendi struktuurist, heteroaatomitest ja funktsionaalsetest rühmadest. Mitme teguri koosmõju korral on tundlikkuse määramine analoogia põhjal piiratud ja ebatäpne. Katsed kolonni ja detektori vahele asetatud kuumutatud toruga näitasid, et temperatuur avaldab tugevat mōju detektori tundlikkusele. Võib arvata, et kıumade gaasikanalite olemasolu detektoris on üheks pōhjuseks, miks erinevate detektoritega saadud katsetulemused on sageli erinevad.

Esitatakse puhtuse määramise metoodika tundmatute lisandite korral.

\section{P. LUIGA, R. PUSS}

\section{QUANTITATIVE DETERMINATION OF IMPURITIES IN ORGANIC COMPOUNDS WITH FLAME-IONIZATION DETECTOR}

An evaluation of experimental conditions that must be considered in quantitative impurity determinations in the $10^{-2}+10^{-6}$ range with the Shandon FB-4 gas chromatograph is given, with particular attention to flame parameters (gas velocities, electrode voltages) and detector linearity. As the specific response of impurities is not always. known, the relationships between detector response and molecular structure are important. The dependence of detector response to molecular structure, especially to various functional groups has been investigated for a variety of compounds. It has been shown that even moderate thermal cracking between the column and the flame has a profound effect upon the detector response. A practical prescription for impurity determination with flame ionization detectors is given. 